\title{
Validity of Point-of-Care Testing Mission Plus in Detecting Anemia
}

\author{
Noor Ani Ahmad*, MBBS, MPH; S Maria Awaluddin, MD; Rahama Samad, RN; \\ Noraida Mohd Kasim, RN; Muslimah Yusof, RN; Mohd Aznuddin Abd Razak, BSc; \\ Chan Ying Ying, MMedSc; Norhafizah Sahril, BSc
}

\author{
Institute for Public Health, Ministry of Health Malaysia, Kuala Lumpur, Malaysia
}

\begin{abstract}
Background: Point-of-care testing, POCT, was widely used to assess hemoglobin status before proceeding with the confirmation test. Our study aimed to assess the validity of Mission ${ }^{\circledR}$ Plus $\mathrm{Hb}$ in detecting hemoglobin levels in a general population compared with a standard laboratory hematology analyser as the gold standard.

Methods: Two types of samples, capillary and venous blood, were collected from all respondents by trained nurses. Both blood samples were tested using Mission® Plus while the remaining venous blood in EDTA test tubes was sent to the reference laboratory.

Results: A total of 622 respondents participated in this study, $75 \%$ of them females. The mean $\mathrm{Hb}$ tested from capillary blood using Mission ${ }^{\circledR}$ Plus $\mathrm{Hb}$ was $11.80 \pm 2.02 \mathrm{~g} / \mathrm{dl}$. For venous blood, the mean $\mathrm{Hb}$ concentrations using Mission ${ }^{\circledR}$ Plus $\mathrm{Hb}$ and Sysmex XE-2100 hematology analyser were $12.16 \pm 1.84 \mathrm{~g} / \mathrm{dl}$ and $13.07 \pm 1.87 \mathrm{~g} / \mathrm{dl}$, respectively. Sensitivity and specificity in detecting anemia from venous samples were $98.8 \%$ and $73.4 \%$ respectively. Positive Predictive Value (PPV) was 58.5\%, while Negative Predictive Value (NPV) was 99.4\%.

Conclusion: The findings of moderate PPV should alert the programme managers to the importance of a confirmatory test following screening using Mission $\AA$ Plus $\mathrm{Hb}$.
\end{abstract}

Keywords: anemia; point-of-care testing (POCT); Mission ${ }^{\circledR}$ Plus Hb.

\section{Introduction}

Anemia affects populations of both developed and developing countries. In 2008, WHO estimated that anemia affected 1.62 million people worldwide, with estimated prevalence of $30.2 \%$ among non-pregnant women above 15 years of age [1]. In the Western Pacific region, it is estimated that $21.5 \%$ of non-pregnant women are anaemic [1]. In Malaysia, there is a lack of data on the national prevalence of anemia among the general population of non-pregnant women. A regression-based estimate by WHO reported that $30.1 \%$ of non-pregnant women aged 15 to 49.9 years of age were anaemic [1]. Several local studies estimated that the prevalence ranges from $17.2 \%$ [2] among non-pregnant women in interior Sarawak to $34.6 \%$ to $42.3 \%$ among pregnant women $[3,4]$.

*Corresponding author: NoorAni Ahmad. Institute for Public Health, Ministry of Health Malaysia, Jn Bangsar, Kuala Lumpur, Malaysia.E-mail:drnoorani@moh.gov.my
Anemia is considered to be a moderate public health problem when the prevalence is more than $20 \%$ [1].

Various methods have been used to detect anemia in the population at risk. The methods range from hemoglobin colour scale, Sahli technique, copper-sulphate method, HaemoCue, and automated haematology analysers [5]. HemoCue was considered to be the method of choice for POCT due to its reliability, portability, and ease of use [6-9]. But taking into account the economic implication, a less costly but still reliable POCT should be looked into to be used in a nationwide survey to estimate the prevalence of anaemia in Malaysia. In Malaysia, assessment of hemoglobin level using Mission ${ }^{\circledR}$ Plus $\mathrm{Hb}$ has been used in several primary-care settings without proper validation. As it is, a scientific validity test is urgently needed to ensure the validity of this POCT in detection of anemia.

Our study aimed to assess the validity of Mission ${ }^{\circledR}$ Plus $\mathrm{Hb}$ in detecting hemoglobin levels in a general population compared with a standard laboratory hematology analyser (Sysmex XE-2100) as the gold standard. 


\section{Methods}

This is a cross-sectional study involving outpatient respondents recruited from a suburban primary care clinic in Selangor. A minimum sample size of 600 was required, based on $30 \%$ estimated prevalence of anaemia, with $90 \%$ sensitivity and specificity, using formulae for the sensitivity and specificity studies [10]. The study was reviewed and approved by the Medical Ethics and Research Committee, Ministry of Health Malaysia (NMRR-13-797-17207). Mission ${ }^{\circledR}$ Plus Hb (Acon Laboratories, USA) provided an in-kind Hemoglobin Meter and strips for the study.

The eligible respondents voluntarily participated in this study. The eligibility criteria were that participants must be 15 years of age or older and have never been diagnosed as having any blood disorders. Written consents were taken from all respondents. They were informed of the results of their blood tests, and respondents with anemia were referred to a family physician at the clinic for further management.

Data were collected using quota sampling in July 2014. Capillary and venous blood samples were collected from all respondents by trained nurses. A drop of venous blood directly from the needle was then tested with Mission Plus and its reading was documented. The remaining venous blood was then transferred into a test tube containing potassium EDTA as anticoagulant and tested using Sysmex XE-2100 at the reference laboratory within 2 hours. Capillary blood from a similar respondent was then taken from the fingertip and directly tested using Mission Plus. For capillary testing, care was taken to remove the first drop of blood with a sterile cotton swab, and pressure on the finger that might result in hemodilution was avoided. The blood sample was taken using pipette supplied together with the strip. The blood was then dropped on the strip connected to the hemoglobin meter. Its reading was then documented. The Mission Plus used in this study was calibrated daily and the procedure was supervised by a representative from the manufacturer to minimise human error.

The hemoglobin concentration of venous blood using Mission ${ }^{\circledR}$ Plus $\mathrm{Hb}$ was compared to the hemoglobin concentration of venous blood using the gold standard procedure (Sysmex XE-2100). Sysmex XE-2100 was selected as the gold standard in this study based on its accuracy and precision in detecting anemia $[11,12]$. Venous and capillary blood readings using Mission Plus were then compared to determine the accuracy of the test using capillary blood in detecting anemia. Anemia was defined as $\mathrm{Hb}$ less than $12 \mathrm{gm} /$ $\mathrm{dl}$ for women above 15 years of age and $\mathrm{Hb}$ less than $13 \mathrm{gm} / \mathrm{dl}$ for men above 15 years of age [1].

The sensitivity, specificity, predictive positive and predictive negative values were then calculated using the following formulae:

Sensitivity $=\mathrm{TP} /(\mathrm{TP}+\mathrm{FN})$

Specificity $=\mathrm{TN} /(\mathrm{TN}+\mathrm{FP})$

Positive Predictive Value, $\mathrm{PPV}=\mathrm{TP} /(\mathrm{TP}+\mathrm{FP})$

Negative Predictive Value, NPV $=\mathrm{TN} /(\mathrm{TN}+\mathrm{FN})$

Accuracy $=(\mathrm{TP}+\mathrm{TN}) / \mathrm{n}$,

where $\mathrm{TP}$ is the number of respondents correctly identified as anaemic by Mission Plus; FN is the number of respondents identified as not anaemic by Mission ${ }^{\circledR}$ Plus $\mathrm{Hb}$ but noted to be anaemic using Sysmex; FP is the number of participants identified as anaemic using Mission Plus but not anaemic using Sysmex; TN is the number of participants correctly observed to be not anaemic using Mission Plus; and $\mathrm{n}$ is the number of participants. Correlation between the results based on the Mission Plus in comparison to those tested using the gold standard were calculated. Pearson's correlation coefficient and paired t-test were used to analyse the data using IBM SPSS Statistics (IBM Corporation, New York, USA).

\section{Results}

A total of 622 respondents participated in this study, $75 \%$ of them females. Age ranged from 15 to 77 years $(35.7 \pm 12.9$ years). The results obtained from Mission $\AA$ Plus $\mathrm{Hb}$ and the standard reference method are summarised in Table 1.

Table 1.

Hemoglobin $(\mathrm{Hb})$ values of respondents $(n=622)$

\begin{tabular}{lccc}
\hline \multicolumn{1}{c}{ Test } & & $\begin{array}{c}\mathrm{Hb}(\mathrm{g} / \mathrm{dl}) \\
\text { mean } \pm \mathrm{SD}\end{array}$ & $\begin{array}{c}\text { Range } \\
(\mathrm{min}-\mathrm{max})\end{array}$ \\
\hline Mission ${ }^{\circledR}$ Plus Hb & Capillary & $11.80 \pm 2.02$ & $6.4-19.8$ \\
& Venous & $12.16 \pm 1.84$ & $5.5-20.1$ \\
Gold standard (reference) & Venous & $13.07 \pm 1.87$ & $6.9-21.3$ \\
Sysmex XE-2100 & & & \\
\hline
\end{tabular}

On average, the $\mathrm{Hb}$ concentration using capillary and venous samples tested using Mission Plus were both lower than the level using Sysmex, a difference of $-1.27 \mathrm{~g} / \mathrm{dl}(95 \%$ CI: $-1.34,-1.21)$ and $-0.911 \mathrm{~g} / \mathrm{dl}(95 \% \mathrm{CI}:-0.96,-0.86)$, respectively.

Overall, 169 respondents $(98.8 \%)$ were observed to be truly anaemic based on a venous sample using Mission Plus. Based on the capillary results, 166 respondents (97.1\%) were identified as anaemic using Mission Plus, while five respondents were falsely identified as anaemic. Table 2 shows the sensitivity, specificity, PPV, NPV and accuracy of detecting anaemia using Mission Plus.

Table 2.

Validity of Mission Plus in detecting anemia in comparison to standard reference

\begin{tabular}{lcc}
\hline \multicolumn{1}{c}{ Validity } & Anemic* (venous) & Anemic* (capillary) \\
\hline Sensitivity (95\% CI) & $98.8 \%$ & $97.1 \%$ \\
Specificity (95\% CI) & $73.4 \%$ & $62.5 \%$ \\
Positive Predictive Value & $58.5 \%$ & $49.6 \%$ \\
Negative Predictive Value & $99.4 \%$ & $98.3 \%$ \\
Accuracy & $80.4 \%$ & $72.8 \%$ \\
\hline *Hb $<12$ g/dl for females and $\mathrm{Hb}<13 \mathrm{~g} /$ dl for males
\end{tabular}

The Pearson's correlation coefficient between Mission ${ }^{\circledR}$ Plus $\mathrm{Hb}$ and standard reference was high; for capillary hemoglobin the correlation, r, was $0.909(P<0.001)$, while for venous haemoglobin, r was $0.940(P<0.001)$. 


\section{Discussion}

In a community survey, the collection of venous blood samples is mostly unacceptable and the analysis of fresh blood at the standard laboratory is unfeasible. The anemia estimation using POCT allows the collection of information that may otherwise be inaccessible. In order for Mission Plus to be an acceptable POCT for haemoglobin measurement in a nationwide survey, it would have to be precise and accurate. The Ministry of Health Malaysia has produced a guideline to safeguard patient safety and management [13].

Our study found that the $\mathrm{Hb}$ concentrations in the capillary and venous samples tested with Mission ${ }^{\circledR}$ Plus $\mathrm{Hb}$ were on average lower than those recorded in the reference laboratory using venous samples. The standard for assessing the accuracy of the $\mathrm{Hb}$ measurement using POCT varies based on its clinical usage; it ranges from $0.5 \mathrm{~g} / \mathrm{dl}$ for a perioperative blood transfusion [14] to $1.0 \mathrm{~g} / \mathrm{dl}$ in various other settings $[6,15]$. As such, a mean difference of $1.27 \mathrm{~g} / \mathrm{dl}$ for capillary and $0.91 \mathrm{~g} / \mathrm{dl}$ for venous samples using Mission ${ }^{\circledR}$ Plus $\mathrm{Hb}$ as compared to the standard reference is considered good. The $\mathrm{Hb}$ estimation using Mission Plus as the screening tool in a national survey should be interpreted with caution as it may result in overestimating the prevalence of anaemia in Malaysia. The possibility that humidity may change the reagents in the Mission ${ }^{\circledR}$ Plus $\mathrm{Hb}$ hemoglobin meter should also be considered, as happened in a validity study of POCT HemoCue in Australia [16]. Thus, maintenance of proper storage and handling is very crucial.

The findings of lower $\mathrm{Hb}$ using POCT Mission ${ }^{\circledR}$ Plus $\mathrm{Hb}$ did not agree with findings from POCT HemoCue, which exhibited higher $\mathrm{Hb}$ compared to the results from the automated analyser [9,17-20]. Technical errors such as incomplete filling or bubbles in a pipette may have also influenced the results from some samples in our study.

Our study found that the ability of the POCT to detect anaemia, based on the PPV, was moderate. In addition, the specificity of the Mission Plus in detection of non-anaemia was $62.5 \%$ and $73.4 \%$ for capillary and venous samples, respectively. This figure is lower compared to a specificity of $100 \%$ using POCT HemoCue $[9,17,18]$. In contrast, the sensitivity of the Mission Plus for screening for anaemia was almost $100 \%$ for both capillary and venous samples. Furthermore, the correlation was also high. Thus, considering the above-mentioned values, Mission Plus is suitable to be used as a screening method for detection of anaemia in a mass population although it is necessary to emphasize a thorough training of those involved in the survey to minimize technical errors.

Unlike other POCTs that have high specificity but moderate sensitivity, we found high sensitivity with moderate specificity and accuracy of Mission Plus in the detection of anaemia. The reasons for these differences require further investigation. Further study is also required to assess the conversion factor from capillary to venous blood at different levels of anemia.

Our study has several strengths. Our samples included the whole spectrum of age groups from young adults to the elderly to avoid selection bias. We also used a blind and independent comparison between the findings using Mission ${ }^{\circledR}$ Plus $\mathrm{Hb}$ at a primary care centre with the measurement of haemoglobin using Sysmex XE-2100 at the reference laboratory. Our sample size was properly calculated using a specific formula and was much bigger compared to the sample size of other validity studies of a POCT. A limitation noted from this study is the lack of comparison with arterial blood samples. Other validation POCT studies have compared capillary with venous and arterial blood samples, but in our study, collection of arterial blood samples was not suitable as the study site was a primary care centre.

\section{Implications and recommendations}

A highly sensitive tool is crucial for a screening, but high specificity is also a desired criterion to avoid unnecessary false positive results. As such, a positive finding with this POCT must be followed up by a confirmatory test before starting on any treatment. This tool might not be suitable to be used in a clinical setting such as monitoring of anaemic patients. On the positive side, we support the use of this POCT for screening for anemia in a mass population due to its characteristics as a rapid, fairly accurate and less costly method compared to other POCTs. However, it is necessary to note that any POCT should not replace formal laboratory venous sampling, which still remains the gold standard for haemoglobin measurement.

\section{Conclusion}

Our study found that Mission ${ }^{\circledR}$ Plus $\mathrm{Hb}$ has high sensitivity and moderate specificity and is considered suitable to be used as a screening tool for assessment of hemoglobin levels in a mass population.

\section{Competing interests}

The authors declare that they have no competing interests.

\section{Acknowledgements}

The authors would like to thank the Director General of Health Malaysia for his permission to publish this article.

We thank Dr Nazrila Hairizan and her staffs at Pandamaran Health Clinic for collecting blood samples for the study.

We acknowledge Dr Wan Hayati Wan Yusof and her staffs at Hospital Tengku Ampuan Rahimah Klang for processing and analysis of all venous blood samples. We thank Dr Tahir Aris, Director of the Institute for Public Health Malaysia for his support in conducting this study.

\section{References}

1. Worldwide prevalence of anaemia 1993-2005: WHO global database on anaemia. Edit by De Benoist B, McLean E, Egli I, Cogswell M. World Health Organization, Geneva, 2008. 2. Sagin DD, Ismail G, Mohamad M, Pang EK, Sya OT. Anemia in remote interior communities in Sarawak, Malaysia. 
Southeast Asian J Trop Med Public Health 2002; 33(2): 373-7. 3. Hassan R, Abdullah WZ, Nik Hussain NH. Anemia and iron status of Malay women attending an antenatal clinic In Kubang Kerian,Kelantan, Malaysia. Southeast Asian J Trop Med Public Health 2005; 36(5):1304-7.

4. Thirukkanesh S, Zahara AM. Compliance to Vitamin and Mineral Supplementation among Pregnant Women in Urban and Rural Areas in Malaysia. Pakistan J Nutrition 2010; 9(8):744-750.

5. Srivastava T, Negandhi H, Neogi SB, et al. Methods for hemoglobin estimation: a review of "What Works". J Hematol Transfus 2014; 2(3):1028.

6. Sanchis-Gomar F1, Cortell-Ballester J, Pareja-Galeano H, Banfi G, Lippi G. Hemoglobin point-of-care testing: the HemoCue system. J Lab Autom 2013; 18(3):198-205.

7. Neufeld L, García-Guerra A, Sánchez-Francia D, NewtonSánchez O, Ramírez-Villalobos MD, Rivera-Dommarco J. Hemoglobin measured by Hemocue and a reference method in venous and capillary blood: a validation study. Salud Publica Mex 2002; 44(3):219-27.

8. Paiva Ade A, Rondó PH, Silva SS, Latorre Mdo R. Comparison between the Hemocue and an automated counter for measuring hemoglobin. Rev Saude Publica 2004; 38(4):585-7.

9. Shahshahani HJ, Meraat N, Masouri F. Evaluation of the validity of a rapid method for measuring high and low haemoglobin levels in whole blood donors. Blood Transfus 2013; 11(3):385-90.

10. Sample size calculation for sensitivity and specificity studies. http://www.docstoc.com/docs/168682724/Samplesize-for-sensitivity-_-specificity-studies-_by-Lin-Naing (accessed on January $\overline{5}, 2015$ ).

11. Walters J, Garrity P. Performance Evaluation of the
Sysmex XE-2100 Hematology Analyzer. Lab Hematol 2000; 6:83-92.

12. Tsuruda K, Tsuji T, Usui T, et al. Evaluation and Clinical Usefulness of the Automated Hematology Analyser, Sysmex XE-2100. Sysmex J Int 1999; 9(2):129-138.

13. National Point of Care Testing: Policy and Guidelines. Departmental Policy of Pathology Services, Ministry of Health Malaysia, 2012.

14. Gehring H, Hornberger C, Dibbelt L, Rothsigkeit A, Gerlach K, Schumacher J, et al. Accuracy of point-of-caretesting (POCT) for determining haemoglobin concentrations. Acta Anaesthesiol Scand 2002; 46(8):980-6.

15. Agarwal R, Heinz T. Bedside hemoglobinometry in hemodialysis patients: lessons from point-of-care testing. ASAIO J 2001; 47(3):240-3.

16. Nguyen HT. High humidity affects HemoCue cuvette function and HemoCue haemoglobin estimation in tropical Australia. J Paediatr Child Health 2002; 38(4):427-8.

17. Mendrone A Jr1, Sabino EC, Sampaio L, Neto CA, Schreiber GB, Chamone Dde A, et al. Anemia screening in potential female blood donors: comparison of two different quantitative methods. Transfusion 2009; 49(4):662-8.

18. Akhtar K, Sherwani RK, Rahman K, Hasan J, Shahid M.Hemocue photometer: a better alternative of haemoglobin estimation in blood donors? Indian J Hematol Blood Transfus 2008; 24(4):170-2.

19. Bahadur S, Jain S, Jain M. Estimation of hemoglobin in blood donors: a comparative study using hemocue and cell counter. Transfus Apher Sci 2010; 43(2):155-7.

20. Cable RG, Steele WR, Melmed RS, Johnson B, Mast AE, Carey PM, et al. The difference between fingerstick and venous haemoglobin and hematocrit varies by sex and iron stores. Transfusion 2012; 52(5):1031-40. 